\title{
Mathematical Study of the Verhulst and Gompertz Growth Functions and Their Contemporary Applications
}

\section{Cemil İskender ${ }^{1}$}

\begin{abstract}
This study examines the mathematical characteristics of the logistic, the generalized logistic and the Gompertz growth function used in human population analysis. When a population growth is mathematically modeled, it starts with differential equations considered as a preliminary study. Then, a general solution equation is derived. This is the method followed by the mathematicians who developed these models. To prepare for the study, I used the framework of the objectives and adhered to the resources and approaches outlined by mathematicians who developed the growth function. In addition, I wanted to evaluate the methodologies that remain valid in contemporary applications using the current perspectives. Mathematician and actuary Benjamin Gompertz developed the first survivors' function in 1825 which was used later as a population growth function while systematizing life tables. In his three published articles, the mathematician Pierre-François Verhulst developed a logistic human population growth function based on his economic analysis. In addition, he searched for test opportunities using the limited population statistics of France, Belgium, England, the USA, and Russia. Contemporary authors Richards and, 'Ricketts and Head' made very invaluable contributions to logistic growth function.
\end{abstract}

\section{Keywords}

Verhulst logistic function, Gompertz growth function, Generalized logistic growth function, Differential equations of Growth functions

1 Corresponding author: Cemil i̇skender (Retired Research Economist), Istanbul, Turkey. E-mail: iskenderc@ttmail.com ORCID: 0000-0003-2841-5964

To cite this article: Iskender, C. (2021). Mathematical Study of the Verhulst and Gompertz Growth Functions and Their Contemporary Applications. EKOIST Journal of Econometrics and Statistics, 34, 73-102. https://doi.org/10.26650/ekoist.2021.34.876749 


\section{Introduction}

This study examines the mathematical properties (including the historical development) of the Verhulst (logistic) growth function, generalized logistic growth function, and Gompertz growth function used in human population analysis. I have emphasized the properties of mathematical functions based on the analysis of the development of the human population only. Although there are a large number of growth functions, here I have included those that I have achieved successful application results in my previous statistical studies (İskender 2018 \& 2019). Another point we take into account is that the authors of the growth functions that I examined used extensive statistical data to prove their theory. As an economist and statistician, this point impressed me, and I have felt close to these authors. Growth of human population is an important subject matter of economic sciences, for example as explained by Malthus and Verhulst more than two centuries ago. Many functions have been developed outside the human population for the analysis of biomedical sciences, botanical, and zoological events and communities; however, these disciplines are completely outside the scope of this article even if the logistic and Gompertz functions are used by authors in these fields very frequently.

When a population growth is modeled, it starts with differential equations that can be characterized as a preliminary study, and the derivation of a general solution equation is found. This is the method followed by the early mathematicians who developed these models. The resulting general solution equation is applied to the population data with statistical software and parameters, and other related findings of the equation are determined. In the final stage, it is possible to make predictions using these functions. The practice and predictions of statistical applications will not be covered in this article.

Based on the above objectives and using the resources and approaches of the mathematicians who developed growth functions, I evaluated the methodologies that remain valid until today in the contemporary works. Also, I took advantage of the approaches of the subsequent authors who contributed to the growth problem. I considered bringing together the perspectives of the mathematicians who were pioneers in the research area with today's advanced statistical applications as applied in my previous works (İskender, 2018, 2019). In the meantime, I wanted to investigate the problems in the process of their historical development. Therefore, I started first by determining the differential equations and included research by Verhulst and Gompertz who had been ignored in the past and the contributions of whom had not been considered for over a century, yet still valid.

In the framework described above, the three articles of Verhulst $(1838,1845,1847)$, allometric coefficient $(1 / v)$ of Richards (1959), second curvature with weight function of 'Ricketts and Head' (1999) that I see as main contributions will be discussed for 
the logistic growth function. Also, the theoretical introduction of Gompertz's (1825) article and Sprague's (1861) explanations for the Gompertz differential to general solution equation for Gompertz growth function and of course addition of lower asymptote $(L)$ for both Verhulst's and Gompertz's function will be next discussion subjects. Although there are many other growth function studies, none of them will be subject to review here.

\section{Logistic Growth Function of Verhulst}

The articles on population written by the Belgian professor of mathematics PierreFrançois Verhulst (1804-1849) are "Notice on the Law That the Population Follows in Its Increase" (1838), "Mathematical Researches on the Law of Population Growth" (1845) and "Second Work on the Population Growth Law" (1847), respectively (Verhulst, 1838, 1845, and 1847). Verhulst described his function as logistic, and he named exponential curve as logarithmic. From the twentieth century onward, there has been a revival of interest in long-forgotten writings of him; subsequently, the function was called logistic and have been used until today. When I consider the titles that I provide above and the article contents, I should also need to qualify the function as mathematical population growth law. I understand that Verhulst developed and used the word "logistic" to mean "meets geographical and environmental conditions and constraints". I also believe that the word logistic is used in comparison with (prorata) Malthus's "logarithmic" curve.

When Verhulst begins working on the progress of population, his approach in the first sentence of his article written in 1845 defines the basis of event from a political economy point of view:

"Of all the problems that political economy offers to the meditations of philosophers, one of the most interesting is, without a doubt, the knowledge of the law that regulates the progress of the population. To solve it accurately, one would have to be able to appreciate the influence of the numerous causes that prevent or promote the multiplication of the human species." (Verhulst, 1845, p. 3)

Mathematician Verhulst also had the qualification of an economist like Malthus. Although he is a professor of mathematics, he also has extensive knowledge of his contemporary sciences. In addition, it is understood from the sentence that the progression of population is an issue that philosophers should consider in their work. Being a philosopher means being familiar with many disciplines in Verhulst's age. In his three articles on the population, Verhulst is understood to be an economist firstly. After that, like today's methods, mathematics and statistics will come into play in proving and solving the proposed economic problem. Three of his four published articles are on the human population and statistics, and one is on mathematics. 
When his friend Mr. Quetelet requested him to write an article on human population growth for his book Mathematics and Physics Correspondence, Verhulst complied; he began his article with Malthus's human population analysis and continued with population growth/scarce resources approach of economic theory, he wrote:

"It is known that the famous Malthus has established as a principle that the human population tends to grow in geometric progression, so as to double after a certain period, e.g., every twenty-five years. This proposal is indisputable, apart from the ever-increasing difficulty of obtaining subsistence when the population has acquired a degree of agglomeration, or the resources that the population draws from its growth, even when society is still in its infancy, such as a greater division of labor the existence of regular government and defenses that ensure public tranquility, etc." (Verhulst, 1838, p. 113)

Verhulst, believed that the population will face scarce resources with development, he also highlighted the restrictive effect of public burden on the economic development of society in the early stages of development. The first finding of Verhulst is the wellknown population and limited resources dilemma of economic analysis. The field of economics would possibly need another hundred years to address this issue.

The second phase of his economic analysis is the law of diminishing returns and population relationship. Each increase has a limit. He wrote as follows:

"In our old European societies, where fertile [productive] land has been cultivated for a long time, the work of improving land already in cultivation can only add to its products ever-decreasing quantities; admitting that, in the first twenty-five years, the soil product has been doubled, in the second period only the second period may be able to produce an extra third. The virtual increase in population thus finds a limit in the extent and fertility of the country, and the population tend, therefore, more and more to become stationary." (Verhulst, 1838, p. 113)

Although the country has the potential to create new population growth through foreign trade when its facilities are used to the fullest, this is limited, and the maximum rule of population growth needs to be adhered to, as stated below-quoted text:

"There is a limit to the growth of that population to the extent permitted by the land that meets the need for a population's housing. The fact is that when a population consumes the cultivation opportunities of land, it can gain reciprocal responses from other countries and a new increase in population. But it is clear that these imports will have limits, this situation can happen even long before all the areas of the country are reached. Therefore, all formulas that will try to represent the laws of the population must accept the requirement that maximum can be reached in an extremely distant period. This maximum will be a population that has become stable.” (Verhulst, 1838, pp. 114-115) 
When I evaluate the quotes, it is obvious that Verhulst based his analysis on an economic thought: Certain factors limit the infinite increase rule. Once he established the Mathematical Population Theory, the statistician Verhulst performed application studies as well ${ }^{1}$. In Verhulst, economics, mathematics, and statistics coexisted. However, unlike Malthus's population research that has been used in every stage of economic history to date, Verhulst's research has not been applied to social sciences until the twentieth century or even twenty-first century. In one sense, Verhulst has not yet taken his rightful place, especially in the literature of economics and statistics. Blaug (1978, pp. 69-79), the contemporary writer of economics, does not mention Verhulst when describing Malthus on population theory section of his book.

The mathematical model that Verhulst developed about two hundred years ago carried the same principles as the basis of contemporary approaches, and the basis of the approach has been preserved to date by subsequent writers. The population growth model can be expressed as the difference between the population increase and decrease. Births and deaths are in an organic relationship with the current population; therefore, I can express the increase and decrease in the death and birth rates as a function of the population. Relationships, such as births/population $=f$ (population) and deaths/population $=f($ population $)(f$ : function $)$, lie between the ratios and the absolute numbers. Assuming that these relationships are linear for simplicity, I may define the following functional expressions:

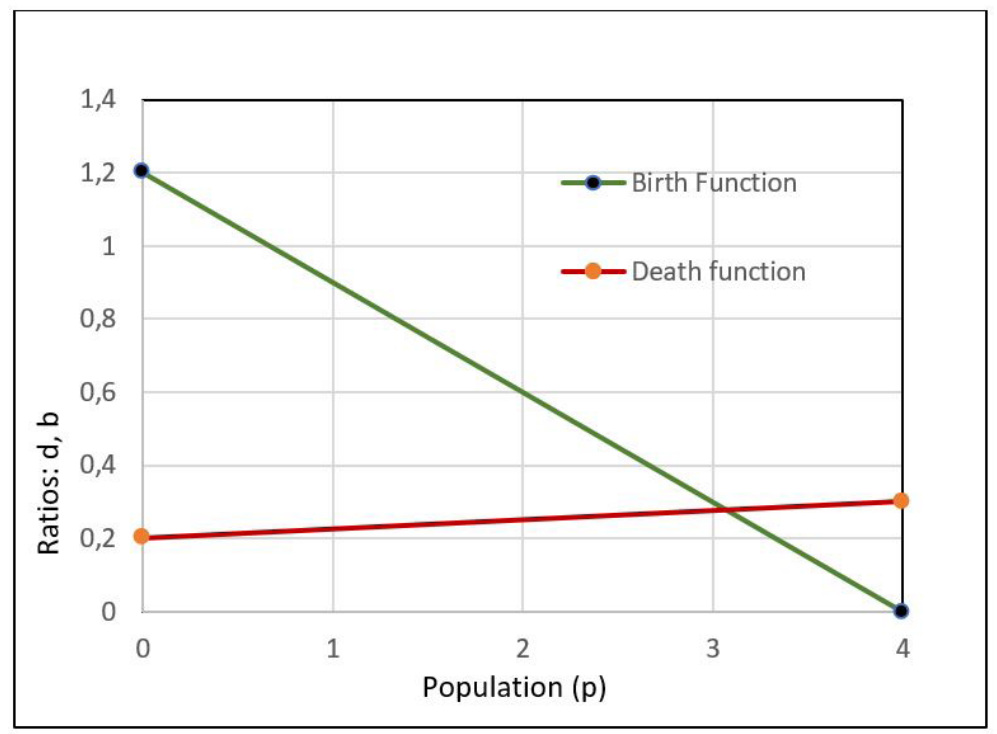

Figure 1. Sample Birth and Death Functions

1 Verhulst writes: "I have been trying for a long time to determine by analysis, the probable law of the population; but I have abandoned this kind of research, because there is too little observational data for the formulas to be verified, so as not to leave any doubt as to their accuracy." (Verhulst, 1838, p. 115). He is well aware of the limited quality of data that he used. 
The death rate $(d)$ and the birth rate $(b)$ can be written as a linear function of the population $(p)^{2}$ as follows ${ }^{3}$ :

$$
\begin{aligned}
& d=\varphi(p)=d_{0}+a p \\
& b=\varphi(p)=b_{0}-c p
\end{aligned}
$$

where $d_{0}, \mathrm{a}, \mathrm{b}_{0}$, and c are constants:

The death rate is an increasing function of the population and increases in parallel with the population growth starting (at least) at $d_{0}$. The coefficient (slope) that determines the increment rate is $a$. Due to limited resources, ${ }^{4}$ the factors detrimental to population growth gain in strength and limit the growth. The birth rate is a decreasing function of the population. It starts to decline from the highest level of $b_{0}$. The coefficient (negative slope) that determines the decrease rate is $c$. The population reaches its highest level at the intersection point of the two curves.

If I define the change in population as the difference between the births and deaths, the differential equation of the model can be given as follows:

$$
\begin{gathered}
\frac{d p}{d t}=b p-d p \\
\frac{d p}{d t}=p\left(b_{0}-c p\right)-p\left(d_{0}+a p\right)
\end{gathered}
$$

where $p$ is the population, and $t$ is the time.

By replacing the definitions of $m=b_{0}-d_{0}$ and $n=c+a$ and making necessary arrangements, the differential equation can be given as follows:

$$
\begin{gathered}
\frac{d p}{d t}=p b_{0}-c p p-p d_{0}-a p p \\
\frac{d p}{d t}=p\left(b_{0}-d_{0}\right)-p p(c+a) \\
\frac{d p}{d t}=m p-n p^{2}
\end{gathered}
$$

2 I am tracking Verhulst's notation.

3 Equation order numbers are in parantheses.

4 Verhulst says that Quetelet, who was a mathematician, faculty member, and his colleague, was thinking that these forces were inversely proportional to the square of the population multiplied by the sum of the gradients of the birth and mortality functions (). Verhulst's differential equation is also built on this idea. (Verhulst, 1838) 
In this way, I obtained the differential equation of Verhulst. The parameter $m$ is the difference between the maximum birth rate and minimum mortality rate at zero time. It also specifies the difference between the intrinsic growth rate of the differential equation and the solution equation. The two curves intersect at the point where the maximum population is $p=\frac{b_{0}-d_{0}}{c+d}$.

If I integrate the differential equation ${ }^{5}$

$$
-\frac{\log _{e}(n p-m)-\log _{e} p}{m}=t+c
$$

and substitute the initial values as $t=0$ and $p=p_{0}$ (the initial point of the population), I have

$$
\frac{\log _{e}\left(\frac{p}{n p-m}\right)}{m}=\frac{m t+\log _{e}\left(\frac{p_{0}}{p_{0} n-m}\right)}{m}
$$

If I solve for $p$, then I obtain the Verhulst ${ }^{6}$ logistic function as follows:

$$
p=\frac{p_{0} m e^{m t}}{p_{0} n e^{m t}-p_{0} n+m}
$$

For $t=+\infty, p=m / n$. In Verhulst's notation lower-case $p$ is the current population. When $t=-\infty$, we have $p=0$.

Dividing the numerator and denominator of (10) by $n$ and instead of lower-case ( $p$ ) substituting upper-case $P=m / n$ yields a logistic population growth function that contains the upper limit of the population as $P$.

$$
p=\frac{p_{0} P e^{m \tau}}{p_{0} e^{m t}-p_{0}+P}
$$

Verhulst first emphasized that the factors that slow down the population growth are an unknown of $p$. Then, the function of $\varphi(p)$ was used as the simplest hypothesis in his article. He assumed that the appropriate provision was $\varphi(p)=n p^{2}$. Verhulst stated that he had also tried the below-given functions and population figures calculated from these functions, and the census results were approximately the same:

5 All the logarithmic expressions in our article are $e$-based natural logarithms, unless otherwise stated.

6 There are no solution details of the differential equation in the article written by Verhulst in 1838. Verhulst uses $\mathrm{p}^{\prime}$ for the initial point of population instead of $p_{0}$. 


$$
\varphi(p)=n p^{2}, \varphi(p)=n p^{3}, \varphi(p)=n p^{4}, \varphi(p)=n \log (p)
$$

The results of the main solution equations that I have calculated according to these four alternatives differ from one another. It is impossible to say that the estimated values calculated by each function give appropriate and close results with the actual observation values. Verhulst might have been expressing the idea that predictions calculated with the term of $\varphi(p)=n p^{2}$ were almost the same as the actual observation values.

When I take the death function as $\varphi(p)=n p^{l}$ and solve the differential equation, the solution equation was found to be $P=P_{0} e^{t(m-n)}$. This form is the well-known exponential growth function. Verhulst did not mention this simple exponential case in his writing. Replacing the term of death as $\varphi(p)=n p^{2}$ in the equation, I obtained the growth function of (11). When the growth function is replaced by $\varphi(p)=n p^{3}, \mathrm{I}$ obtained two solution equations and when y $\varphi(p)=n p^{4}$, I obtained three solution equations but two of them contains imaginary numbers.

Verhulst reached a solution by defining the upper limit $P$ of the population as the upper asymptote in his mathematical model. In the application of growth models with statistics, there is a need for defining the lower asymptote and including it in the model. Researchers, Paine et al. (2012) have used mathematical models, including the lower asymptote level $(L)$. I also preferred the use of two asymptote models in my previous studies (İskender, 2018, 2019) because of the possibilities provided by these models.

I define the following variables:

Y Population, dependent variable,

$t \quad$ Time, explanatory variable,

$T \quad$ Base year ${ }^{7}$,

$K \quad$ Upper asymptote, at most level of population

$L \quad$ Lower asymptote, at least level of population

$p_{0}=Y_{0} \quad$ Initial population,

$m=r \quad$ Intrinsic rate of growth,

$p=Y-L \quad$ Difference between the current population level and lower asymptote,

$P=K-L \quad$ Difference between the upper and lower asymptotes,

$7 \mathrm{~T}$ and $\mathrm{t}$ were used differently. $\mathrm{T}$ is the base year, and $\mathrm{t}$ is time, explanatory variable. 
Substituting these variables in (11), yields

$$
Y-L=\frac{Y_{0}(K-L) e^{r(t-T)}}{Y_{0} e^{r(t-T)}-Y_{0}+(K-L)}
$$

Dividing the numerator and denominator by $Y_{0} e^{r(t-T)}$, I obtain

$$
Y-L=\frac{K-L}{1+\frac{\left(-Y_{0}+(K-L)\right)}{Y_{0} e^{r(t-T)}}}
$$

If I define and replace $Q=\frac{\left(-Y_{0}+K-L\right)}{Y_{0}}$, I obtain the final growth function as follows:

$$
Y=L+\frac{K-L}{1+\frac{Q}{e^{r(t-T)}}}
$$

or as:

$$
Y=L+\frac{K-L}{1+Q e^{-r(t-T)}}
$$

Here, $Y$ is the population (a dependent variable), and $t$ is the time (an explanatory variable) indexed with the base year $T$. The logistic function is a mathematical growth function with two variables, four parameters $K, L, Q$, and $r$ are to be estimated and has a nonlinear structure. The non-linearity definition of the function refers to the structure in which other parameters are found in the derivative equations when the partial derivatives of the function are taken according to each parameter successively.

There are no four separate simultaneous functions at hand to determine the four parameters. Therefore, a statistical analysis of nonlinear functions requires special software that contains convergence criterion and the iteratively weighted leastsquares estimation. NLIN procedure of SAS ${ }^{\circ}$ Studio software provides linearity tests in addition to known (standard errors, F-test etc.) statistical tests: Hougaard's skewness, Box's bias test and Ratkovsky's global nonlinearity measures are among them. For details of nonlinearity and references see (Gebremariam, 2014; SAS Institute Inc., 2017, 2018).

According to SAS/STAT® 14.3 User's Guide:

"The NLIN procedure fits nonlinear regression models and estimates the parameters by nonlinear least squares or weighted nonlinear least squares... Estimating parameters in a nonlinear model is an iterative process that commences from starting values. You need to declare the parameters in your model and supply their initial values for the 
NLIN procedure... Nonlinear least-squares estimation involves finding those values in the parameter space that minimize the (weighted) residual sum of squares." (SAS Institute Inc., 2017, p. 6616)

The summary description I give just above regarding the statistical application of logistic function also applies to generalized form of logistic and Gompertz functions.

Allen $^{8}(1969$, p. 419) prefers to use the differential equation based on upper limit value of population $(K)$ for the solution equation of the logistic function. To do this, I write the differential equation of Verhulst as follows:

$$
\begin{gathered}
\frac{d p}{d t}=m p-n p^{2} \\
\frac{d p}{d t}=m p-\frac{m}{m} n p p=m p\left(1-\frac{n p}{m}\right)=m p\left(1-\frac{p}{m / n}\right)
\end{gathered}
$$

and replace the definitions $K=m / n, m=r$ and $p=Y$

$$
\frac{d Y}{d t}=r Y\left(1-\frac{Y}{K}\right)
$$

I re-write the equation as follows:

$$
\frac{K d Y}{Y(K-Y)}=r d t
$$

Integrating both sides:

$$
\int \frac{K d Y}{Y(K-Y)}=r t+C
$$

I can write the left-hand side of (17) according to the partial fraction method as follows:

$$
\int \frac{K d Y}{Y(K-Y)}=\int\left(\frac{1}{Y}+\frac{1}{K-Y}\right) d Y=\log _{e} Y-\log _{e}(K-Y)=-\log _{e} \frac{K-Y}{Y}
$$

8 British economist, statistician and mathematician Sir Roy G. D. Allen lived between 1906-1983, was lecturer at the London School of Economics and wrote many books on mathematical economics. 
Therefore,

$$
\log _{e} \frac{K-Y}{Y}=-r t-C
$$

After rearranging and replacing $Q=e^{-C}$ (an arbitrary constant), I obtain

$$
\frac{K-Y}{Y}=e^{-r t} e^{-C}=Q e^{-r t}
$$

and reach the following logistic equation:

$$
Y=\frac{K}{\left(1+Q e^{-r t}\right)}
$$

With the addition of the lower asymptote $L$ and the base year $T$, I obtain (15) as solution equation.

The mathematical properties that are useful for the population analysis of the Verhulst logistics growth function are as follows:

Logistic function of Verhulst:

$$
Y_{(t-T)}=L+\frac{K-L}{1+\mathrm{Q} e^{-r(t-T)}}
$$

The first derivative of the function shows the absolute growth (that is the change increase or decrease) in years:

$$
\frac{d}{d(t-T)}\left[Y_{(t-T)}\right]=\frac{(K-L) \mathrm{Q} r e^{-r(t-T)}}{\left(\mathrm{Q} e^{-r(t-T)}+1\right)^{2}}
$$

I calculate the relative growth rate or the annual average growth, by dividing the first derivative figure with the value of the function:

$$
\frac{\frac{d}{d t}\left[Y_{(t-T)}\right]}{Y_{(t-T)}}=\frac{(K-L) \mathrm{Q} r e^{-r(t-T)}}{\left(Q e^{-r(t-T)}+1\right)^{2}\left(\frac{(K-L)}{\mathrm{Q} e^{-r(t-T)}+1}+L\right)}
$$

The second derivative of the function is used to calculate the inflection points of the curve and to monitor changes in the absolute growth:

$$
\frac{d^{2}}{d(t-T)^{2}}\left[Y_{(t-T)}\right]=\frac{2(L-K) Q r^{2} e^{-2 r(t-T)}}{\left(Q e^{-r(t-T)}+Q\right)^{3}}-\frac{(L-K)(Q) r^{2} e^{-r(t-T)}}{\left(e^{-r(t-T)}+Q\right)^{2}}
$$


Time inflection point of the function: When the second derivative is equated to zero and solved for $(t)$, I determine the inflection point of time as follows:

$$
t_{\text {inf }}=\frac{r T+\log _{e}[Q]}{r}
$$

or

$$
t_{\text {inf }}=T+\frac{1}{r} \log _{e}[Q]
$$

If I replace the time inflection point in (23), I find the population figure in the middle of the logistic function as follows between the lower and upper asymptotes:

$$
Y=\frac{K+L}{2}
$$

The logistic function has a symmetrical structure. It has a turning point in the middle of the curve. At this point, the relative growth is at the highest level. The logistic curve also brings some problems with its symmetrical structure. Thus, when the function is symmetrical, the course of the first and second half of the curve follows the concave/convex frame. If the actual figures of the analyzed population are not close to a symmetrical structure, then the ability to represent the logistics curve is decreased. The authors who noticed this problem have developed the methods that I will consider in section 3 .

$$
\operatorname{Lim}_{t \rightarrow \infty} Y=K \text { and } \operatorname{Lim}_{t \rightarrow-\infty} Y=L .
$$

The differential equation of (15) with the upper and lower asymptotes is as follows:

$$
\frac{d(Y-L)}{d t}=r(Y-L)\left(\frac{K-Y}{K-L}\right)
$$

\section{Generalized Logistic Growth Function}

\subsection{First Development}

The logistic growth function was developed by writers in the twentieth century while expanding the application areas and new requirements. The first of these developments was seen in the article of Richards F. J., (1959). Here, the author proposed that $1 / v(1 /(1-\mathrm{m})$ in his manuscript) be located at the denominator exponent of the logistic function.

As the title of the Richards F. J., (1959) article suggests, the variable $v$ provides 
flexibility to the predicted dependent variable of the function and increases the compliance of the curve to the actual data. The authors specified $v$ as the "allometric coefficient." When $v=1$, it becomes the logistic growth function of (15). A logistic function given in the form of (31) is called a "Richards logistics function or curve".

Function:

$$
Y_{(t-T)}=L+\frac{K-L}{\left(1+Q e^{-r(t-T)}\right)^{1 / v}}
$$

First derivative of the function:

$$
\frac{d}{d(t-T)}\left[Y_{(t-T)}\right]=\frac{(K-L) \mathrm{Q} r e^{-r(t-T)}\left(\mathrm{Q} r e^{-r(t-T)}+1\right)^{-\bar{v}-1}}{\mathrm{~V}}
$$

Annual average growth (or relative growth rate); the first derivative is divided by the function:

$$
\frac{\frac{d}{d t}\left[Y_{(t-T)}\right]}{Y_{(t-T)}}=\frac{(K-L) \mathrm{Q} r e^{-r(t-T)}\left(\mathrm{Q} r e^{-r(t-T)}+1\right)^{-\frac{1}{v}-1}}{\mathrm{v}\left(\frac{(K-L)}{\left(\mathrm{Q} e^{-r(t-T)}+1\right)^{1 / v}}+L\right)}
$$

Second derivative of the function:

$$
\frac{d^{2}}{d(t-T)^{2}}\left[Y_{(t-T)}\right]=\frac{(L-K) Q r^{2} e^{-r(t-T)}}{v\left(Q r e^{-r(t-T)}+1\right)^{\frac{1}{v}}\left(e^{-r(t-T)}+Q\right)^{2}}-\frac{(L-K) Q^{2} r^{2} e^{-r(t-T)}\left(Q e^{-r(t-T)}+1\right)^{-\frac{1}{v}-1}}{v^{2}\left(e^{-r(t-T)}+Q\right)}
$$

Time inflection point of the function is as follows:

$$
t_{i n f}=\frac{r T+\log _{e}\left[\frac{Q}{v}\right]}{r}
$$

I find population figure equivalence of the inflection point as follows:

$$
Y=\frac{L(v+1)^{1 / v}-L+K}{(v+1)^{1 / v}}
$$

The structure of the curve is asymmetrical. $\mathrm{Y}$ is not equivalent to $(K+L) / 2$. The flexibility of adaptability is high because $v$ affects the formula. Therefore, it is quite different from the logistic curve.

$$
\operatorname{Lim}_{t \rightarrow \infty} Y=K \text { and } \operatorname{Lim}_{t \rightarrow-\infty} Y=L \text {, and } Y \text { is calculated from the function. }
$$


The differential equation of the logistic growth function which is based on $(v)$ parameter and the details of obtaining the solution equation may be found in Heinen (1999) and Skiadas (2010).

\subsection{Second Development}

The second important modification of the logistic growth function was the application of authors Ricketts and Head (1999). The aim is to strengthen the asymmetric structure of the logistic function.

The authors separated $Q$ (i.e., starting value, multiplier of $(e)$ base of the growth function) into two multipliers $Q_{1}$ and $Q_{2}$. Also, the weighting function as weighted with the harmonic mean $Q_{1}$ and $Q_{2}$ of was included in the equation defining $f_{t}$ for $Q_{1}$ and $\left(1-f_{t}\right)$ for $Q_{2}$. They made the symmetry of the logistic function asymmetrical. The $Q e^{-r(t-T)}$ term of the logistic equation is as follows in the generalized logistic growth function:

$$
Q e^{-r(t-T)} \equiv f_{t} Q_{1} e^{(-r(t-T))}+\left(1-f_{t}\right) Q_{2} e^{(-r(t-T))}
$$

When $Q_{1}$ becomes equal to $Q_{2}$, I obtain a simple logistic function. However, the authors made a different application by changing the location of some parameters as required by the nature of the medical event that they examined. In their study, they took the logistic growth function of (15) as their base; there was no $1 / v$ parameter in its denominator (See İskender, 2018 for details).

They also applied the weighting function of (41) to the coefficients in the denominator of logistic function. It "defines a logistic weighting function varying smoothly between 0 and 1" (Ricketts \& Head, 1999, p. R444).

Let $Y$ be the population (dependent variable) and $t$ be the time (explanatory variable) indexed with the base year T. The generalized logistic growth function is a mathematical function with two variables and six parameters $\left(K, L, Q_{1}, Q_{2}, r, v\right)$; this function has a nonlinear structure. The various mathematical properties of the functions are as follows:

Generalized logistic growth function:

$$
Y_{(t-T)}=L+\frac{K-L}{\left(1+f_{t} A+\left(1-f_{t}\right) B\right)^{(1 / v)}}
$$

Coefficients:

$$
A=Q_{1} e^{(-r(t-T))}
$$




$$
B=Q_{2} e^{(-r(t-T))}
$$

Weighting function:

$$
f_{(t-T)}=\frac{1}{1+\bar{C}_{f} e^{(-r(t-T))}}
$$

Harmonic mean:

$$
\bar{C}_{f}=\frac{2 Q_{1} Q_{2}}{\left|Q_{1}+Q_{2}\right|}
$$

If I write the functions of (38) - (42) together, the generalized logistic function takes the following broad and detailed form?

$$
Y_{(t-T)}=L+\frac{K-L}{\left(1+\frac{1}{1+\frac{2 Q_{1} Q_{2}}{\left|Q_{1}+Q_{2}\right|} e^{(-r(t-T))}} Q_{1} e^{(-r(t-T))}+\left(1-\frac{1}{1+\frac{2 Q_{1} Q_{2}}{\left|Q_{1}+Q_{2}\right|} e^{(-r(t-T))}}\right) Q_{2} e^{(-r(t-T))}\right)^{(1 / v)}}
$$

The weight function, allometric coefficient $v$ and separation of $Q$ into two multipliers as $Q_{1}$ and $Q_{2}$. overcome the constraints imposed by the symmetry event and to better reflect the asymmetrical nature of the data; therefore, the inflection point was not in the middle of the curve. The flexibility of this function is as much an advantage as it is, one and most important drawback is the increase in the number of parameters to be estimated by two more. Difficulties of estimating of the increased number of parameters are felt during the computer software application. In summary; I defined the logistic population growth function as the generalized logistics growth function based on changes; (i) addition of lower asymptote $(L)$, (ii) the addition of the allometric coefficient ( $1 / v)$, (iii) the separation of the coefficient $(Q)$ of base $(e)$ into two (as $Q_{1}$ and $Q_{2}$ ), (iv) the inclusion of the weighting function $f_{(t-T)}$ in the denominator of equation.

Although the computer outputs of the mathematical properties of the generalized logistic function (43), like first derivative, relative growth rate and second derivative formulas etc. are easily available but voluminous, I have not included them here to keep this article short.

\section{Gompertz's Survivors Function or Population Growth Function of Gompertz}

In the first quarter of the 19th century, the British, self-educated, mathematician and actuary Benjamin Gompertz (1779-1865), in his work on the mortality tables,

9 It is more convenient to use the above partitioned equations (38)-(42) in computer software, such as SAS Studio Software, Microsoft Excel, and so on. For applications of a wide range of functions including growth functions in the SAS environment, see (Sit \& Poulin-Costello, 1994). 
made the following assertions. This excerpt is taken from Gompertz's article of 1825 , as it was without any changes, and reflects the writing style of that period:

"I now call the reader's attention to a law observable in the tables of mortality, for equal intervals of long periods; and adopting the notation of my former paper, considering $L_{x}$ to express the number of living at the age $x$, and using $\lambda$ for the characteristic of the common logarithm; that is, denoting by $\lambda\left(L_{x}\right)$ the common logarithm of the number of persons living at the age of $x$, whatever $x$ may be, I observe that if $\lambda\left(L_{\chi}\right)-\lambda\left(L_{n+m}\right)$, $\lambda\left(L_{n+m}\right)-\lambda\left(L_{n+2 m}\right), \lambda\left(L_{n+m}\right)-\lambda\left(L_{n+3 m}\right), \& \mathrm{c}$. be all the same; that is to say, if the differences of the logarithms of the living at the ages $n, n+m ; n+m, n+2 m ; n+m, n+3 m ; \&$ c. be constant, then will the numbers of living corresponding to those ages form a geometrical progression; this be the fundamental principle of logarithms. This law of geometrical progression pervades, in an approximate degree, large portions of different tables of mortality." (Gompertz, 1825, p. 514)

In the life tables, the sample population (cohort size), which is a certain value at the age of zero, will change because of deaths ${ }^{10}$. The changes in the sample population are equal to the rate of mortality multiplied by the initial population volume. According to Gompertz's observations, the sample population series will change geometrically by age; that is the essence of the assumption. The change will be geometrical; therefore, it is possible to determine this change in a mathematical form. But geometric progression in life tables applies to both survivors and deaths. A geometric decrease is seen in survivors, and a geometric increase is seen in deceased persons.

In his study, Gompertz based his observation on the fact that when the differences of logarithms of the survivors' figures are arranged equally, the number of survivors by age (which coincide with these equal intervals) would indicate a geometric decrease. In his calculations on four life tables that he analyzed, the number of survivors in the life table decreased geometrically when the logarithms of the survivors' figures in the life table were ordered at equal intervals. To explain our goal diagrammatically, I drew the graph shown in Figure 2 from the Northampton life table in Gompertz's article. The chart shows the equidistant logarithmic values of the population on the vertical axis, and the population quantities corresponding to these values are on the horizontal axis. A visual examination of the chart shows that the curve has a geometric progression.

10 See İskender (2019) for a detailed application and perfect combination of Gompertz curve and Richards logistic curve with Turkish life tables data of 2015 and explanations of life tables generally. 


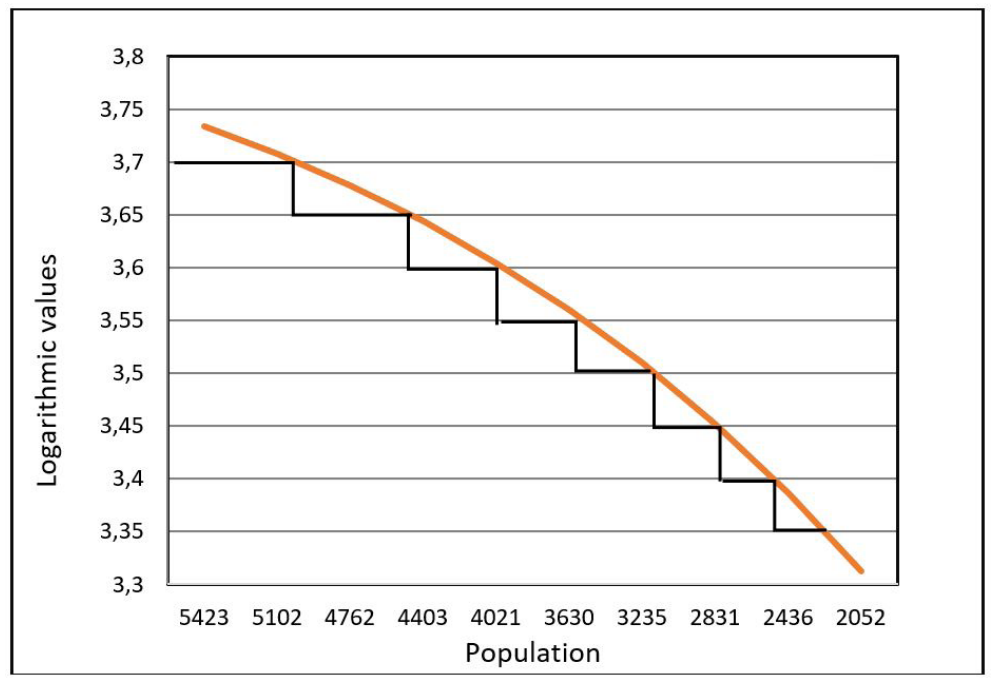

Figure 2. Life Table Graph of Northampton

Note: Compiled from Northampton life table of Gompertz, 1825

Starting with the assumption that logarithmic differences are constant, I determine the age and population sequences that coincide with these fixed intervals in life tables, which is a very labor-intensive task. The ages that coincide with equal logarithmic intervals will be determined by trial and error. Even if the equality of the logarithmic differences in the middle-age groups of the life tables occurs as expected, this rule can deviate at the upper-end and lower-end regions of the curve. The life tables examined by Gompertz generally covered the population between the ages of 10-60 years. Those were the some additional problems that complicate Gompertz's research.

For a more precise approach, I rearranged the Northampton mortality table as a pivot table (table 1) and a graph (figure 3). I have specified equal age intervals on the horizontal axis of the figure and the natural logarithms of equal age intervals of survivors on the vertical axis. When the table and graph are examined, as the equal age intervals on the horizontal axis increase, the logarithmic differences in the vertical axis also increase in geometric order; this means that the number of survivors decrease rapidly. The increase in the logarithm numbers of the equal age intervals suggest that the number of deaths increase geometrically. Here, the decisive explanatory variable is the age progression. A geometric decrease in the survivors' figures also means a geometric increase in the number of deaths. Therefore, the Gompertz function can also be defined and evaluated as a growth function (Winsor, 1932) \"On the Nature of the Function Ex- pressive of the Law of Human Mortality, '" in which he showed that I"if the average exhaustions of a man's power to avoid death were such that at the end of equal infinitely small intervals of time, he lost equal portions of his remaining power to oppose destruction, ," then the number of sur- vivors at any age $\mathrm{x}$ would be 
given by the equation LX $=$ kge (1. However, I aim to follow the first explanation of the growth function herein through the descending series of life tables.

Table 1

Northampton Mortality Table of Gompertz (Summary) ${ }^{11}$

\begin{tabular}{lcccc}
\hline Age & Survivors & $\begin{array}{c}\text { Logarithms of } \\
\text { Survivors }\end{array}$ & $\begin{array}{c}\text { Logarithmic Differences } \\
\text { (Five-year) }\end{array}$ & $\begin{array}{c}\text { Logarithmic Differences } \\
\text { (Ten-year) }\end{array}$ \\
\hline 15 & 5423 & 3.73424 & -0.02650 & \\
20 & 5102 & 3.70774 & -0.02995 & \\
25 & 4762 & 3.67779 & -0.03404 & -0.06399 \\
30 & 4403 & 3.64375 & -0.03941 & -0.08384 \\
35 & 4021 & 3.60433 & -0.04443 & \\
40 & 3630 & 3.55991 & -0.05003 & -0.10797 \\
45 & 3235 & 3.50987 & -0.05793 & \\
50 & 2831 & 3.45194 & -0.06526 & -0.13976 \\
55 & 2436 & 3.38668 & -0.07450 & \\
60 & 2052 & 3.31218 &
\end{tabular}

Note: Compiled from Northampton mortality table of Gompertz, (1825).

The Northampton pivot table ${ }^{12}$ and the graph I have arranged reveal a summary of the theory. In the population analysis, I explain using logarithmic scales because I am interested in proportional changes, not absolute changes (Allen, 1969, pp. 220-221, 246). In the table and graph, the geometrical growth of the differences between the logarithms of the surviving population according to equal age intervals requires the study of the $m, m p, m p^{2}, m p^{3}$, which is based on a differential equation of the series of structures.

In the first part of Gompertz's article, the solution of the differential equation was based on logarithmic calculations, although the basis of the assumption was a geometric series. In the second presentation, Gompertz followed a method that was directly based on a geometric series for solving the differential equation.

11 I use dot to separate the decimal places in my article.

12 Gompertz supported his theoretical model by working on the four life tables: Northampton, Deparcieux, Sweden, and Carlisle. Last three tables whose graphs are not shown in this article also show curves similar to that of the Northampton table. 


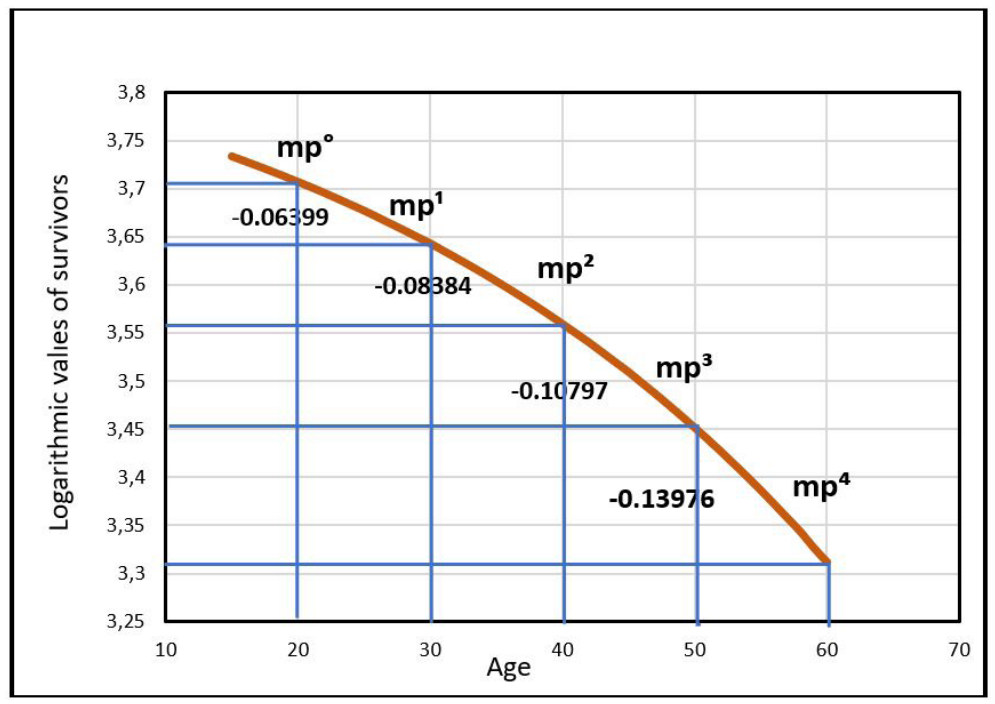

Figure 3. Graph of Northampton Table (Ten-year basis)

\subsection{Basic Solution of Gompertz}

In his first approach, Gompertz gives the solution of the differential equation in six steps of logarithmic definitions. This excerpt is taken from Gompertz's article of 1825 , as it was, without any changes, and reflects the writing style of that period. As I explained below, it carries many printing errors. (The equation order numbers in the circles mine)

"If the average exhaustions of a man's power to avoid death were such that at the end of equal infinitely small intervals of time, he lost equal portions of his remaining power to oppose destruction which he had at the commencement of those intervals, then at the age $x$ his power to avoid death, or the intensity of his mortality might be denoted by $a q^{x}$, $a$ and $q$ being constant quantities; and (1) if $L_{x}$ be the number of living at the age $x$, we shall have $\mathrm{a} L_{x} \times q^{x} \cdot \dot{x}$ for the fluxion of the number of deaths $=-\left(L_{x}\right)$; (2) $\therefore a b q^{x}=\frac{L_{x}}{L_{x}}$, (3) $\therefore a b q^{x}=$ - hyp. $\log$. of $b \times$ hyp. $\log$. of $L_{x}$, and (4) putting the common logarithm of $\frac{1}{b} \times$ square of the hyperbolic logarithm of $10=c$, (5) we have $c . q^{x}=$ common logarithm of $\frac{L_{x}}{d}$ ; d being a constant quantity, and (6) therefore $L_{x}$ or the number of persons living at the age of $x=d \cdot g\urcorner q^{x}$; $g$ being put for the number whose common logarithm is $c$. The reader should be aware that I mean $g^{7 q^{x}}$ to represent $g$ raised to the power $g^{x}$ and not $g^{q}$ raised to the $x$ power; which latter I should have expressed by $g^{7 q^{x}}$, and which would evidently be equal to $g^{\mathrm{u}^{x}}$. I take this opportunity to make this observation, as algebraists are sometimes not sufficiently precise in their notation of exponentials"13 (Gompertz, 1825, p. 518)

The first sentence describes the two dimensions of the assumption, that is, the power to avoid death (survivors) and the remaining power to oppose destruction or

13 The $\mathrm{x}$ 's in the excerpt refer to multiply and $x$ to age of survivors. Three triangle dots $(\therefore)$ mean 'therefore'. Fluxion: derivative. Hyp. log.: natural logarithm. 
the rate of approach to the end (deaths). The function expresses both states: survivals and fatalities. In other words, the curve represents a series that is both decreasing and ascending, depending on whether the sign is negative or positive. However, it is painful for mathematicians to follow and understand the explanation and achieve the solution equation using the six steps described in the second part of the sentence. The mathematical descriptions are very short; the critical auxiliary equations of the solution are not in the text, and there are printing errors. ${ }^{14}$ In addition, the sentences are very long with a few sentences even spanning half a page. The spellings used by Gompertz are also difficult to follow. ${ }^{15}$ In 1861 , debates started on the article, Sprague (1861) published a work that gave a systematic explanation of the Gompertz's proof and found printing errors as Gompertz was moving from the differential equation to the solution equation in his article. Sprague's explanation is a lengthy alternative, but is easily understandable. ${ }^{16}$

According to Sprague, "If we denote the mortality rate as, where $a$ and $q$ are constants, and if $L_{x}$ is the number of persons living at the age $x$, then the number of deaths in the shortest time interval of $d x$ will be $a L_{x} q^{x}$ ". I can write the differential equation as follows ${ }^{17}$ :

$$
d L_{x}=-a L_{x} q^{x} d x
$$

or as:

$$
\frac{1}{L_{x}} \frac{d L_{x}}{d x}=-a q^{x}
$$

The requirement of the rule ${ }^{18}$

$$
\frac{\mathrm{d}\left(\log _{e} y\right)}{d x}=\frac{1}{y} \frac{d y}{d x}
$$

14 The $b$ 's in the text above will be "x or dot" (meaning multiplication) (Sprague, 1861).

15 The same understanding dominates Adam Smith's famous work "The Wealth of Nations," which was first released in 1776 .

16 Sprague, in his criticism of the time directed at Gompertz, stated that the Royal Society did not allow any corrections in the published articles as a rule. He himself examined the copy that contained Gompertz's corrections. The corrections were made in the Institute of Actuators Library. These corrections were printing errors and did not change any of the content. The author wrote this article to clarify the criticism directed Gompertz. However, he also included a reference to the article of Peter Gray who previously discussed the subjects that he mentioned when he was writing on the mathematical explanation of Gompertz's formula (Gray, 1857).

17 The equation sequence numbers on the left-hand side (1-9) facilitate the follow-up of the equations in Sprague's article.

18 The average variation of a function (i.e., division of a derivative by a function) is equal to the derivative of the logarithm of that function. (Allen, 1969, p. 246) 
yields:

$2 a$

$$
\frac{\mathrm{d}\left(\log _{e} L_{x}\right)}{d x}==-a q^{x}
$$

If I integrate both sides, $\log _{e} D$ being the integral constant ${ }^{19}$,

$$
\log _{e} L_{x}=-\frac{a q^{x}}{\log _{e} q}+\log _{e} D
$$

By rearrangement of (48), I may obtain

$$
\begin{array}{r}
\log _{e} L_{x}-\log _{e} D=-\frac{a q^{x}}{\log _{e} q} \\
3 \\
\log _{e} \frac{L_{x}}{D}=-\frac{a q^{x}}{\log _{e} q}
\end{array}
$$

Following the rule $\log _{a} \frac{1}{q}=-\log _{a} q(a$ is any base), I have

$3 \mathrm{a}$

$$
\log _{e} \frac{L_{x}}{D}=\frac{a q^{x}}{\log _{e} \frac{1}{q}}
$$

I substitute the following two equations (52 and 53) in (51).

$3 \mathrm{~b}$

$$
\log _{e} \frac{L_{x}}{D}=\log _{10} \frac{L_{x}}{D} \log _{e} 10
$$

$3 c$

$$
\log _{e} \frac{1}{q}=\log _{10} \frac{1}{q} \log _{e} 10
$$

Then, I obtain

$4 a$

$$
\log _{10} \frac{L_{x}}{D} \log _{e} 10=\frac{a q^{x}}{\log _{10} \frac{1}{q} \log _{e} 10}
$$

By rearranging (54), I obtain

$4 \mathrm{~b}$

$$
\log _{10} \frac{L_{x}}{D}=\frac{a q^{x}}{\log _{10} \frac{1}{q}\left(\log _{e} 10\right)^{2}}
$$

19 I replaced $d$ in Gompertz's final equation with $D$ because $d$ is used to specify the differential (e.g., $d x$ ). 
I define the constant on the right-hand side of (55) as

$$
c=\frac{a}{\log _{10} \frac{1}{q}\left(\log _{e} 10\right)^{2}}
$$

By substituting (56) in (55), I obtain

$$
\log _{10} \frac{L_{x}}{D}=c q^{x}
$$

Exponentiating each side yields the following equality:

$$
\frac{L_{x}}{D}=10^{c q^{x}}
$$

If I replace $g=10^{c}$ or $g=\log ^{-1}{ }_{10}^{c}$, the solution equation is

$$
\frac{L_{x}}{D}=g^{q^{x}}
$$

$$
L_{x}=D g^{q^{x}}
$$

After the differential equation is established in (44), the logarithmic transformation of (47) is used as its base. Thus, the logarithmic differences in the number of survivors are based on a mathematical rule. In the latest phase, we obtain the equation of the general solution with anti-logarithmic arrangements.

The following table lists the equations of Gompertz that also carry printing errors and the corrected and/or updated equivalents of these equations.

Table 2

Gompertz's Equations and Corrected and Updated Equivalents

\begin{tabular}{cccc}
\hline $\mathbf{x}$ & Gompertz's equations & $\mathbf{y}$ & Corrected and $/$ or updated equations \\
\hline 1 & $-\left(\dot{L_{x}}\right)=\mathrm{a} L_{x} \boldsymbol{X} \cdot \dot{x}$ & 1 & $d L_{x}=-a L_{x} q^{x} d x$ \\
2 & $a b q^{x}=-\frac{\dot{L_{x}}}{L_{x}}$ & 2 & $\frac{1}{L_{x}} \frac{d L_{x}}{d x}=-a q^{x}$ \\
3 & $a b q^{x}=-\log _{e} b \boldsymbol{X} \log _{e} L_{x}$ & $2 \mathrm{~b}$ & $\log _{e} L_{x}=-\frac{a q^{x}}{\log _{e} q}+\log _{e} D$ \\
4 & $\log _{10} \frac{1}{b} x\left(\log _{e} 10\right)^{2}=c$ & 5 & $c=\frac{a}{\log _{10} \frac{1}{q}\left(\log _{e} 10\right)^{2}}$ \\
5 & $c \cdot q^{x}=\log _{10} \frac{L_{x}}{d}$ & 6 & $\log _{10} \frac{L_{x}}{D}=c q^{x}$ \\
6 & $L_{x}=x=d \cdot g^{q^{x}}$ & 9 & $L_{x}=D_{g}^{q^{x}}$ \\
\hline
\end{tabular}

Note: The $\mathrm{x}$ column shows the circled numbers of the equations that I marked in Gompertz's excerpt. The y column shows the left-hand-side numbers of the equations of $(44)-(60)$. 
The solution equation can also be achieved by making the following abridged applications in (48). In (48), if we substitute $c=\log (D)$ and $\frac{a}{\log _{e}(q)}=\log _{\mathrm{e}}(g)$ which are logarithmic and constant numbers, we obtain

$$
\log _{e}\left(L_{x}\right)=\log _{e}(D)+q^{x} \log _{e}(g)
$$

Exponentiating both sides yield the final solution:

$$
L_{x}=D g^{q^{x}}
$$

\subsection{Second Solution of Gompertz: Basis of Geometric Series}

The second solution of differential equation in the article (Gompertz, 1825, pp. 519-20, Art. 6.) is based on a geometric progression and arithmetic series. Contrary to the first solution, we find here an explanation that follows the rules of mathematics step-by-step and is also in conformity with the current notation. The solution equation of $L_{x}=a q^{x}$ is appropriate for the Northampton example (table 1, figure 3 ) and is in compliance with the structure of $m, m p, m p^{2}, m p^{3}$ given below.

If be the number of living persons at the age of $x$, we can write the following series as a geometric progression.

$$
\begin{gathered}
\log \left(L_{a}\right)-\log \left(L_{a+r}\right)=m \\
\log \left(L_{a+r}\right)-\log \left(L_{a+2 r}\right)=m p \\
\log \left(L_{a+2 r}\right)-\log \left(L_{a+3 r}\right)=m p^{2}
\end{gathered}
$$

In general, writing the series

$$
\log \left(L_{a+n-r}\right)-\log \left(L_{a+n}\right)=m p^{\frac{n}{r}-1}
$$

by continual addition gives

$$
\log \left(L_{a}\right)-\log \left(L_{a+n}\right)=m\left(1+p+p^{2}+p^{3}+\cdots+p^{\frac{n}{r}-1}\right)
$$

and

$$
\log \left(L_{a}\right)-\log \left(L_{a+n}\right)=m \frac{1-p^{\frac{n}{r}}}{1-p}
$$


Therefore, replacing $q=p^{\frac{1}{r}}$ and $\log (\varepsilon)=\frac{m}{1-p}{ }^{20}$ in (83) with the rule of $\left(p^{\left(\frac{1}{r}\right)}\right)^{n}=(q)^{n}$, we have

$$
\log \left(L_{a+n}\right)=\log \left(L_{a}\right)-\left(1-q^{n}\right) \log (\varepsilon)
$$

and

$$
\log \left(L_{a+n}\right)=\log \left(L_{a}\right)-\log (\varepsilon)+q^{n} \log (\varepsilon)
$$

with the exponential arrangements

$$
\log \left(L_{a+n}\right)=\log \left(\frac{L_{a}}{\varepsilon}\right)+q^{n} \log (\varepsilon)
$$

and

$$
L_{a+n}=\left(\frac{L_{a}}{\varepsilon}\right) \varepsilon^{q^{n}}
$$

Substituting a $+n$ by $x$, we have

$$
L_{x}=\left(\frac{L_{a}}{\varepsilon}\right) \varepsilon^{q^{-a} q^{x}}
$$

Again, by using $D=\frac{L_{a}}{\varepsilon}$ and $g=\varepsilon^{q^{-a}}$, we obtain

$$
L_{x}=D g^{q^{x}}
$$

In our opinion, Gompertz's solution that we provided above is an educational example that should also be included in contemporary studies on advanced differential calculus and their applications. ${ }^{21}$ Unfortunately, this second approach of Gompertz, to solve the differential equation, has not been mentioned and included in any articles, manuscripts etc. until today.

\subsection{Gompertz Function: Updated and Its Mathematical Properties}

I can obtain (75) by making the necessary substitutions in equation (74). For this, I substitute $q=e^{\log (q)}$ in (74) and obtain $q^{x}=\mathrm{e}^{\mathrm{x} \log (q)}$. Substituting the constant number as $\log (q)=-r$ finally yields $q^{x}=e^{-r t}$. However, I may write $\mathrm{g}=\mathrm{e}^{\log (g)}=e^{-Q}$ as the constant $\log (g)=-Q$. Then, I have:

20 This formula $\left[\log (\varepsilon)=\frac{m}{1-p}\right]$ is in the form of $\left[\log (\varepsilon)=\frac{m}{1-q^{n}}\right.$. $]$ in the original article of Gompertz, I have corrected it above believing it to be a printing error.

21 In the references, I see a Gompertz modeling in which the first part is based on the $m, m p, m p^{2}, m p^{3}$ series and the curve slope of Matsui $(1999,2009)$. For an application of Matsui Gompertz equation to Turkish population data see appendix 1 of İskender (2018, pp. 126-128). 


$$
L_{x}=D e^{-Q e^{-r t}}
$$

If I specify $K$ as the upper asymptote, $Y$ as the population, $Y_{0}$ as the initial population, and $t$ as the time (explanatory variable) indexed with the base year $T$, and add the definitions $L_{x}=Y-Y_{0}, D=K-Y_{0}$, and $x=t-T$ the Gompertz function can be obtained as shown in (76).

The Gompertz function is a mathematical growth function with two variables and four parameters $(K, Y, Q, r)$ and has a nonlinear structure.

The Gompertz function,

$$
Y_{(t-T)}=Y_{0}+\left(K-Y_{0}\right) e^{-Q e^{(-r(t-T))}}
$$

may be written in the form of a division as follows:

$$
Y_{(t-T)}=Y_{0}+\frac{K-Y_{0}}{e^{Q\left[\frac{1}{e^{r t}}\right]}}
$$

The first derivative of the function is:

$$
\frac{d}{d(t-T)}\left[Y_{(t-T)}\right]=\left(K-Y_{0}\right) Q r e^{-Q e^{-r(t-T)}-r(t-T)}
$$

The relative growth rate or annual average growth (first derivative/function) of the function is:

$$
\frac{\frac{d}{d t}\left[Y_{(t-T)}\right]}{Y_{(t-T)}}=\frac{\left(K-Y_{0}\right) Q r e^{-Q e^{-r(t-T)}-r(t-T)}}{\left(K-Y_{0}\right) e^{-Q e^{-r(t-T)}}+Y_{0}}
$$

The second derivative of the function is:

$$
\frac{d^{2}}{d(t-T)^{2}}\left[Y_{(t-T)}\right]=\left(K-Y_{0}\right) Q r\left(Q r e^{-r(t-T)}-r\right) e^{-Q e^{-r(t-T)}-r(t-T)}
$$

The time inflection point of the function is:

$$
t_{i n f}=\frac{r T+\ln (Q)}{r}
$$


If I replace the time inflection point in the function, I get,

$$
Y=e^{-1}\left(K+\left(e^{1}-1\right) Y_{0}\right)
$$

The Gompertz function is asymmetrical, and the value of (81) is not a simple arithmetic mean of $K$ and $Y_{0}$.

In the Gompertz population growth function when $r$ and $\mathrm{Q}$ are positive, the value of the function $(Y)$ tends to the upper asymptote $K$ as the explanatory variable time $t$ tends to positive infinity, and the time tends from negative infinity to $Y_{0}$.

The differential equation, which includes the upper asymptote and initial population point of the function, is as follows:

$$
\frac{d\left(Y-Y_{0}\right)}{d t}=r\left(Y-Y_{0}\right)\left[\log _{e}\left(\frac{K-Y_{0}}{Y-Y_{0}}\right)\right]
$$

\section{Final Comments and Results}

\subsection{Comments}

The mathematicians Verhulst and Gompertz not only theoretically explained the population growth, but they also investigated the testing of the models with statistical data using the mathematical models they developed in the first half of the 19th century.

After establishing the mathematical model of the population growth in his first article, Verhulst performed application studies using the population statistics of France, Belgium, Essex, and Russia of his time. With the French population statistics, the figures predicted by the function were extremely close to the actual numbers. The growth function could be successfully applied. In his short essay, there were no details of how the equation was applied to the population data; no mathematical details were followed while reaching the solution equation from the differential equation. Verhulst developed methods for estimating the function parameters even though this work remained unpublished. He posted it in his article of (Verhulst, 1845).

In his article of 1845 , Verhulst reconsidered the population problem in greater detail and published the results of his studies on the population of the United States of America, France, and Belgium. The land and border changes in Europe due to the imperial wars, population registration systems, and so on created significant statistical problems in the data used for analysis. For determining the computational results of function, he developed a mathematical calculation method of the parameters based on calculating the coordinates of the three equally spaced points on the curve. Using the results in his first two articles, Verhulst rediscussed the population issue in his 
paper of 1847 and worked on the population statistics of Belgium and France with a new population growth function that he had developed (Verhulst, 1847).

The theoretical and practical contributions of his writings are apparent. Verhulst studies were quite advanced for his time. We cannot expect Verhulst to achieve complete excellence with the statistical data collected in the first half of the nineteenth century. Even today, we encounter many problems related to data. Today, we know that the number of growth functions has dramatically increased. Growth functions provide different applications of the results in various events. Even if there are growth functions more generally accepted, and they have found application areas, it is not possible a priori to say that one is superior to another before applying the data.

Gompertz worked on the data from four life tables, and he derived and generalized a mathematical formula from the tables' figures. Then he presented his mathematical findings to the highly developed actuaries' sector in England during that period and used it in his own actuary company. Gompertz's model appeared in the following hundred years of actuarial writings (King, 1902). In 1932, the Gompertz function started to be used as a growth function (Winsor, 1932).

Despite the limited data and insufficient statistical accounting techniques of their period, both authors did not remain confined to the mathematics phase of their research, but they also emphasized the statistical application. The methods they applied form the bases of research even today. They knew the importance of the statistical applicability of the mathematical models that they developed. Their writings focus more on data application to the functions and methods than on the theoretical explanations of the models. In the first article of Verhulst, the general solution equation obtained from the differential equation is very short. However, the problems in the arrangement and mathematical application of the population statistics are more prominent in the writings.

The approaches that both authors applied two hundred years ago still remain valid. In the beginning of the 19th century, modern statistical science required more than a hundred years to develop. Nevertheless, Gompertz and Verhulst continued with the mathematical model-statistical application relationship to the very end. In the second half of the twentieth century, the growth functions of Gompertz and Verhulst took top positions in the list of generally accepted growth functions.

\subsection{Results}

- Verhulst did not set out to establish a simple growth function, as mentioned in countless articles for two centuries.

- He developed a mathematical method to create the application of economic thought that he developed and supported that method with statistical data. These aspects of his works have been neglected to this day. 
- Verhulst was an economist, mathematician and one of the first statisticians in the history of economic sciences.

- Verhulst built his work entirely on needs of human population and limited resources dilemma.

- Gompertz's mathematical model, based on life tables, has come to this day as a growth model and has gained widespread popularity. It is the formula of both decreasing and increasing populations.

- It should be accepted that Gompertz was one of the first statisticians with the statistical application of his mathematical model on four life tables of his time. His statistical study covers sixty-three pages of his article which is seventy pages.

- The contributions of contemporary authors (Richards, 1959; Ricketts \& Head, 1999) to Verhulst's growth function have put advanced opportunities in practice in front of us.

- Verhulst's and Gompertz's mathematical works, which reach the general solution equation from the differential equation, are still the best way to be followed in research today and need to be supported by contemporary applied statistical science methods in application.

- As the growth functions have a nonlinear structure, linearity tests (like skewness, bias, global nonlinearity measures tests, as described in SAS Studio software documents) should be performed during statistical applications in addition to known statistical tests. Ordinary statistical test values, such as standard error, F-test etc. are not enough for accurate evaluation and results. (SAS Institute Inc, 2017).

- Along with growth functions; first derivative, relative growth rate, second derivative and inflection point functions should also be used when explaining population development of a country with statistical analysis (İskender, 2018).

\footnotetext{
Hakem Değerlendirmesi: Dış bağımsız.

Çıkar Çatışması: Yazar çıkar çatışması bildirmemiştir.

Finansal Destek: Yazar bu çalışma için finansal destek almadığını beyan etmiştir.

Peer-review: Externally peer-reviewed.

Conflict of Interest: The author has no conflict of interest to declare.

Grant Support: The author declared that this study has received no financial support.
}

\section{References/Kaynakça}

Allen, R. G. D. (1969). Mathematical Analysis for Economists. London, UK: Macmillan and Co. Ltd. (Original work published in 1938).

Blaug, M. (1979). Economic Theory in Retrospect (Third). Cambridge \& London: Cambridge University Press. (Original work published in 1962). 
Gebremariam, B. (2014). Is Nonlinear Regression Throwing You a Curve? New Diagnostic and Inference Tools in the NLIN Procedure (No. Paper SAS384-2014). Retrieved from https:// support.sas.com/resources/papers/proceedings14/SAS384-2014.pdf

Gompertz, B. (1825). On the Nature of the Function Expressive of the Law of Human Mortality, and on a New Mode of Determining the Value of Life Contingencies. Philosophical Transactions of the Royal Society of London, 115, 513-583. Retrieved from http://www.jstor.org/stable/107756

Gray, P. (1857). On Mr . Gompertz's Method for the Adjustment of Tables of Mortality. The Assurance Magazine, and Journal of the Institute of Actuaries, 7(3), 121-130. Retrieved from https://www.jstor.org/stable/41134787

Heinen, M. (1999). Analytical Growth Equations and Their Genstat 5 Equivalents. Netherlands Journal of Agricultural Science, 47, 67-89. https://doi.org/10.18174/njas.v47i1.479

İskender, C. (2018). Türkiye Nüfus Büyümesi ve Tahminleri: Matematiksel Büyüme Modelleri ve İstatistiksel Analiz ile Kuramsal ve Uygulamalı bir Yaklaşım. Istanbul University Econometrics and Statistics E-Journal, 14(28), 75-141. https://doi.org/10.26650/ekoist.2018.14.28.0004

İskender, C. (2019). Türkiye 2014-2016 Hayat Tablolarında Doğrusal-olmayan Büyüme Fonksiyonlar1 Uygulamas1. Ekoist : Journal of Econometrics and Statistics, 14(29), 151-168. https://doi.org/DOI: https://doi.org/10.26650/ekoist.2018.14.29.0011

King, G. (1902). Text Book of The Principles of Interest, Life Annuities, and Assurances, and Their Practical Application Part II Life Contingencies. London: Charles \& Edwin Layton.

Matsui, K. (1999). Gompertz curve. Retrieved from http://gompertz-matsui.la.coocan.jp/Gompertz/ English.html

Matsui, K. (2009). Gompertzian model. Retrieved from https://www.jstage.jst.go.jp/article/ kanzo/50/6/50_6_324/_article/-char/en

Paine, C. E. T., Marthews, T. R., Vogt, D. R., Purves, D., Rees, M., Hector, A., \& Turnbull, L. A. (2012). How to Fit Nonlinear Plant Growth Models and Calculate Growth Rates: An Update for Ecologists. Methods in Ecology and Evolution, 3, 245-256. https://doi.org/10.1111/j.2041210X.2011.00155.x

Richards F. J. (1959). A Flexible Growth Function for Emprical Use. Journal of Experimental Botany, 10(2), 290-300.

Ricketts, J. H., \& Head, G. A. (1999). A Five-Parameter Logistic Equation for Investigating Asymmetry of Curvature in Baroreflex Studies. American Journal of Physiology - Regulatory, Integrative and Comparative Physiology, 277(2), R441-R454. https://doi.org/10.1152/ ajpregu.1999.277.2.R441

SAS Institute Inc. (2011). SAS/STAT® 9.3 User's Guide The NLIN Procedure (Chapter). In SAS Institute Inc. 2011. SAS/STAT® 9.3 User's Guide. Cary, NC: SAS Institute Inc. Cary, NC, USA: SAS Institute Inc. Retrieved from https://support.sas.com/documentation/onlinedoc/stat/930/ whatsnew.pdf

SAS Institute Inc. (2013). SAS/STAT ${ }^{\circledR} 13.1$ User's Guide The NLIN Procedure. In SAS Institute Inc. 2013. SAS/STAT ${ }^{\circledR} 13.1$ User's Guide. Cary, NC: SAS Institute Inc. Retrieved from https:// support.sas.com/documentation/onlinedoc/stat/131/nlin.pdf

SAS Institute Inc. (2017). SAS/STAT® 14.3 User' s Guide The NLIN Procedure. In SAS Institute Inc. 2017. SAS/STAT® 14.3 User's Guide. Cary, NC: SAS Institute Inc. Retrieved from https:// support.sas.com/documentation/onlinedoc/stat/143/nlin.pdf 
SAS Institute Inc. (2018). SAS/STAT® 15.1 User's Guide The NLIN Procedure. In SAS Institute Inc. 2018. SAS/STAT® 15.1 User's Guide. Cary, NC: SAS Institute Inc. Retrieved from https:// support.sas.com/documentation/onlinedoc/stat/151/nlin.pdf

Sit, V., \& Poulin-Costello, M. (1994). Catalog of Curves for Curve Fitting. Retrieved from https:// www.for.gov.bc.ca/hfd/pubs/docs/bio/bio04.pdf

Skiadas, C. H. (2010). Exact Solutions of Stochastic Differential Equations: Gompertz, Generalized Logistic and Revised Exponential. Methodology and Computing in Applied Probability, 12(2), 261-270. https://doi.org/10.1007/s11009-009-9145-3

Sprague, T. B. (1861). On Mr. Gompertz's Law of Human Mortality, and Mr. Edmonds's Claims to its Independent Discovery and Extension. The Assurance Magazine, and Journal of the Institute of Actuaries, 9(5), 288-295. https://www.jstor.org/stable/41135117

Verhulst, P.-F. (1838). Notice Sur La Loi Que La Population Suit Dans Son Accroissement. In A. Quetelet \& J. G. Garnier (Eds.), Correspondance Mathématique et Physique (Vol. 4, pp. 113-121). Retrieved from https://archive.org/details/correspondancem02belggoog/page/n122/ mode/2up

Verhulst, P.-F. (1845). RECHERCHES MATHÉMATQUES SUR LA LOI D’ACCROISEMENT DE LA POPULATION [Mathematical Researches into the Law of Population Growth Increase]. Nouveaux Mémoires de l'Académie Royale Des Sciences et Belles-Lettres de Bruxelles, 18, 1-41, PDF PP. 15-55. Retrieved from http://www.digizeitschriften.de/dms/resolveppn/?PID=P PN129323640_0018\%7Clog7

Verhulst, P.-F. (1847). DEUXIÈME MÉMOIRE SUR LA LOI D’ACCROISEMENT DE LA POPULATION. Mémoires de l'Académie Royale Des Sciences, Des Lettres et Des Beaux-Arts de Belgique, 20, 1-32, PDF pp. 143-174. Retrieved from http://www.digizeitschriften.de/en/ $\mathrm{dms} / \mathrm{img} /$ ?PID=GDZPPN002492350

Winsor, C. P. (1932). The Gompertz Curve as a Growth Curve. Proceedings of the National Academy of Sciences, 18(1), 1-8. https://doi.org/10.1073/pnas.18.1.1 